\title{
Aryl Hydrocarbon Receptor Modulates the Expression of TNF- $a$ and IL-8 in Human Sebocytes via the MyD88-p65NF-kB/p38MAPK Signaling Pathways
}

\author{
Xiao-Xiao Hou ${ }^{a}$ Guangjie Chen ${ }^{b}$ Amir M. Hossinic Tingting $\mathrm{Hu}^{\mathrm{a}}$

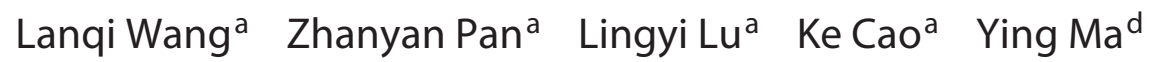 \\ Christos C. Zouboulis ${ }^{c}$ Longqing $\mathrm{Xia}^{\mathrm{a}}$ Qiang Ju ${ }^{\mathrm{a}}$ \\ a Department of Dermatology, Renji Hospital, School of Medicine, Shanghai Jiaotong University, Shanghai, PR China; \\ ${ }^{b}$ Department of Immunology and Microbiology, School of Medicine, Shanghai Jiaotong University, Shanghai, \\ PR China; ${ }^{C}$ Departments of Dermatology, Venereology, Allergology and Immunology, Dessau Medical Center, \\ Brandenburg Medical School Theodore Fontane, Dessau, Germany; ${ }^{\mathrm{d}}$ Department of Dermatology, Huashan \\ Hospital, Fudan University, Shanghai, PR China
}

\section{Keywords}

Peptidoglycan · Acne · p65NF-KB · p38MAPK · Aryl

hydrocarbon receptor $\cdot$ Human SZ95 sebocytes

\begin{abstract}
Activation of Toll-like receptor (TLR)-2 and subsequent inflammatory response contribute to lesion development in acne vulgaris. A cross-talk between aryl hydrocarbon receptor (AhR), a cytosolic receptor protein that responds to environmental and physiological stress, and TLRs has recently been reported. In this study, we explored the possible role of AhR in the effects induced on cultured human SZ95 sebocytes by peptidoglycan (PGN), a classic TLR2 agonist. PGNinduced secretion of inflammatory factors TNF- $a$ and IL-8 in human SZ95 sebocytes was suppressed after knockdown of AhR and pretreatment with the AhR antagonist $\mathrm{CH} 223191$.
\end{abstract}

\section{KARGER}

E-Mail karger@karger.com www.karger.com/jin

\section{(C) 2018 The Author(s)}

Published by S. Karger AG, Basel

This article is licensed under the Creative Commons NonCommercial-NoDerivatives 4.0 International License (CC BYNC-ND) (http://www.karger.com/Services/OpenAccessLicense). Usage and distribution for commercial purposes as well as any distribution of modified material requires written permission.
In addition, the AhR agonist 2,3,7,8-tetrachlorodibenzo- $p$ dioxin (TCDD) enhanced TNF- $\alpha$ and IL-8 secretion in PGNpretreated sebocytes. Furthermore, PGN-induced expression of myeloid differentiation factor 88 (MyD88), phosphop38MAPK (p-p38MAPK), and p-p65NF-KB was strengthened by TCDD and repressed by $\mathrm{CH} 223191$. AhR inhibition by transfecting shRNA blocked the ability of PGN to stimulate phosphorylation of p38MAPK and p65NF-KB in SZ95 sebocytes. Overall, these data demonstrate that $A h R$ is able to modulate PGN-induced expression of TNF- $a$ and IL-8 in human SZ95 sebocytes involving the MyD88-p65NF-kB/ p38MAPK signaling pathway, which probably indicates a new mechanism in TLR2-mediated acne.

\section{(C) 2018 The Author(s)}

Published by S. Karger AG, Basel

X.-X.H. and G.C. contributed equally to this work. 


\section{Introduction}

Skin represents the first-line defense of the human body against external stimuli. The innate immune system is the major contributor to acute inflammation induced by microbial infection and enables the skin to protect the host from infection. In the skin, nonprofessional immune cells, such as keratinocytes and sebocytes, significantly contribute to its innate immunity. Toll-like receptors (TLRs) are the most important pattern recognition receptors, which are responsible for sensing the presence of microorganisms. TLRs, with the exception of TLR3, accomplish their signals through the myeloid differentiation factor 88 (MyD88)-dependent pathway by activating nuclear factor- $\kappa \mathrm{B}(\mathrm{NF}-\kappa \mathrm{B})$ and mitogen-activated protein kinases (MAPKs) and subsequently regulating cytokine gene expression [1].

TLR2 is strongly expressed in human basal and infundibular keratinocytes and sebocytes and is involved in the Cutibacterium acnes-mediated enhancement of acne severity. Exposure of microdissected human sebaceous glands to the pathogen-associated molecular pattern of TLR2 in vitro resulted in an interleukin (IL)-1 $\alpha$ like cornification after 7 days of exposure [2]. Moreover, human sebocytes are immunologically activated by bacterial proteins via TLRs and CD14, which are both expressed on their cell surface [3]. Cell-free extracts of $C$. acnes stimulate cytokine production through activation of TLR in SZ95 sebocytes [4]. Activation of pattern recognition receptors increases the secretion of both proand anti-inflammatory cytokines (chemokines [IL-8], TNF- $\alpha$, antimicrobial lipids, peptides) in sebaceous glands [5-7].

Aryl hydrocarbon receptor $(\mathrm{AhR})$ is a ligand-activated transcription factor that mediates a spectrum of toxic and biological effects of TCDD and related compounds [8]. Recent research has shown that AhR not only plays an important role in toxic action, but also in cell physiology including the human immune system [9]. 2,3,7,8-Tetrachlorodibenzo- $p$-dioxin (TCDD) is a high-affinity AhR ligand that is frequently used to investigate biological processes impacted by AhR activation [10]. In addition, close interactions between TLRs, NF- $\kappa B$, and the AhR signaling pathway have been studied in human dendritic cells [11] and macrophages $[12,13]$, which contribute to immune dysfunction, metabolism of xenobiotics, and carcinogenesis [14].

In this study, we investigated whether peptidoglycan (PGN) could induce activation of the innate immune response, as well as secretion of the proinflammatory fac- tors TNF- $\alpha$ and IL-8 in human SZ95 sebocytes in vitro. Furthermore, we studied the effect of AhR in the innate immune signaling pathway, by knockdown of the AhR gene and applying the AhR agonist TCDD to the cells, to further explore the possible mechanism of the pathogenesis of TLR-mediated acne.

\section{Materials and Methods}

\section{Cell Culture and Reagents}

The immortalized human sebaceous gland cell line SZ95 [15] was routinely maintained in Sebomed ${ }^{\circledR}$ basal medium-Biochrom, Berlin, Germany) supplemented with $5 \mathrm{ng} / \mathrm{mL}$ human epidermal growth factor, $10 \%$ fetal calf serum, $1 \%$ penicillin/ streptomycin (all from Gibco, Invitrogen, Carlsbad, CA, USA), and $1 \mathrm{mM} \mathrm{CaCl}_{2}$ (Sigma, St. Louis, MO, USA) in a humidified atmosphere containing $5 \% \mathrm{CO}_{2}$ at $37^{\circ} \mathrm{C}$. The medium was replaced daily, and cells were used at passage 30-40. Small hairpin RNA (shRNA) targeted against AhR (shAhR) and shCon SZ95 sebocytes were generated according to our previous study [16]. shAhR and shRNA consisting of a scrambled sequence that would not lead to specific degradation of any cellular message (shCon) were generated (GeneChem, Shanghai, PR China). These plasmids were further co-transfected into 293T cells with lentiviral packaging plasmids to generate an AhR shRNA-expressing lentivirus ( $\mathrm{Lv}$-shAhR) or a control shRNA-expressing one (Lv-shCon). SZ95 sebocytes cultured in 6-well plates were infected with titrated viral supernatant at an MOI of 20 in the presence of $5 \mathrm{~g} / \mathrm{mL}$ polybrene (Sigma) to generate AhR-silenced SZ95 cells and controls.

\section{Activation of Cell Cultures}

Cell cultures were grown to approximately $70-80 \%$ confluence and were treated with medium only, PGN $(20 \mu \mathrm{g} / \mathrm{mL}$ ) (from Staphylococcus aureus; Sigma), TCDD (10 nM/L) (Sigma), and CH223191 (10 $\mu \mathrm{M})$ (Tocris Bioscience, Minneapolis, MN, USA). $\mathrm{CH} 223191$ was added in cell medium $1 \mathrm{~h}$ prior to PGN and TCDD. PGN was prepared in culture medium, and TCDD and CH223191 were prepared in DMSO. In PGN-treated cells, medium without the additives to be tested served as control. In TCDD and $\mathrm{CH} 223191$ treatment experiments, DMSO $(0.1 \% \mathrm{v} / \mathrm{v})$ was added as control. Cell counting was conducted using a cell counting chamber slide and cell viability was determined using the MTT assay.

\section{Real-Time RT-PCR}

SZ95 sebocytes were seeded into 24-well plates (Corning) at a density of $1 \times 10^{5}$ cells/well. Total RNA was isolated by Trizol reagent (Invitrogen). RNA concentration was determined at $260 \mathrm{~nm}$ (NanoDrop; Thermo Scientific). Reverse transcript was performed using an mRNA reverse transcription kit (Takara, Tokyo, Japan). The mRNA level of selected genes was measured with SYBR Green (Applied Biosystems ${ }^{\mathrm{TM}}$ ). The primers (Sangon, Shanghai, PR China) used were as follows:

TNF- $\alpha$ forward: $5^{\prime}$-ATGTTGTAGCAAACCCTCAAGC- $3^{\prime}$ and reverse: $5^{\prime}$-GAGGAGCACATGGGTGGAG-3', IL-8 forward: $5^{\prime}$-CCTGATTTCTGCAGCTCTGTG- ${ }^{\prime}$ and reverse: ${ }^{\prime}$-CCAGA-
Hou/Chen/Hossini/Hu/Wang/Pan/Lu/ $\mathrm{Cao} / \mathrm{Ma} /$ Zouboulis/Xia/Ju 


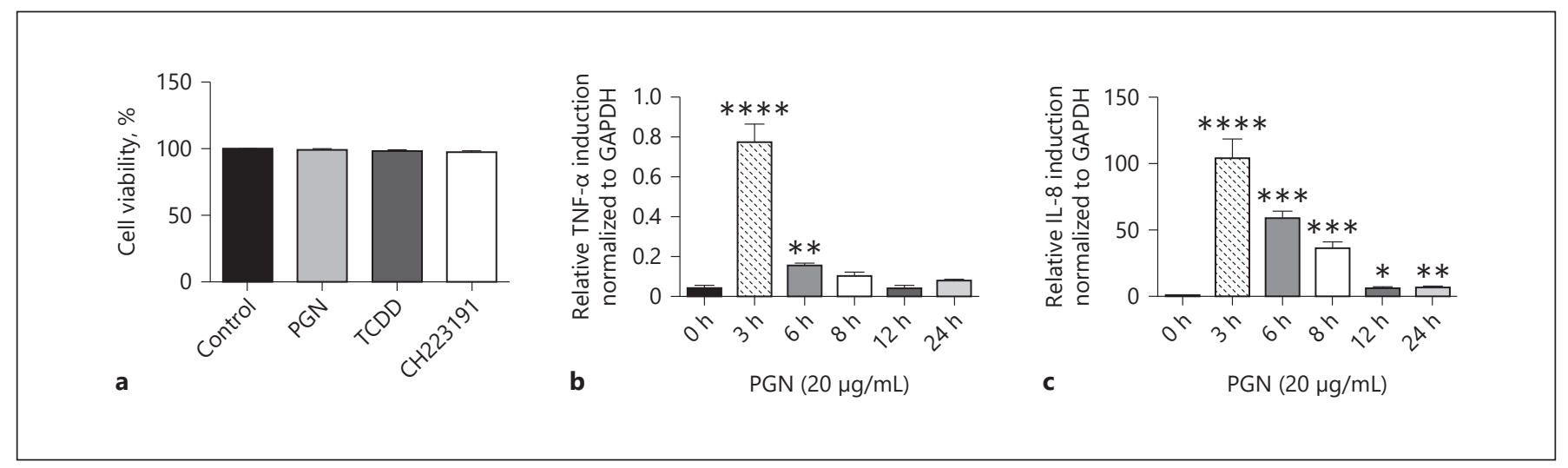

Fig. 1. The effect of PGN, TCDD, and CH223191 on SZ95 sebocyte viability. a After treatment of SZ95 sebocytes with PGN (20 $\mu \mathrm{g}$ / $\mathrm{mL})$, TCDD $(10 \mathrm{nM})$, and $\mathrm{CH} 223191(10 \mu \mathrm{M})$ for $48 \mathrm{~h}$, cell viability was measured by MTT assay. Data (mean \pm SEM) are expressed as a percentage of the control value $(n=6)$. PGN induced mRNA expression of TNF- $\alpha$ and IL-8 in SZ95 sebocytes. Cells were treated for indicated times and relative mRNA levels of TNF- $\alpha(\mathbf{b})$ and IL-8 (c) were assessed by RT-PCR normalized to GAPDH. The results are representative of three independent experiments with similar results. ${ }^{*} p<0.05,{ }^{* *} p<0.01,{ }^{* * *} p<0.001,{ }^{* * * *} p<0.0001$.
CAGAGCTCTCTTCCAT-3', MyD88 forward: 5'-CTGCTCGAGCTGCTTACCAA-3' and reverse: 5'-GATGGGCACCTGGAGAGAGG-3', IL-1 $\alpha$ forward: $5^{\prime}$-GTTTGAGTCAGCAAAGAAGTCAA- $3^{\prime}$ and reverse: $5^{\prime}$-GGAGTGGGCCATAGCTTACAT- ${ }^{\prime}$, CYP1A1 forward: 5'-CACCCTCATCAGTAATGGTCAGA-3 ${ }^{\prime}$ and reverse: $5^{\prime}$-AACGTGCTTATCAGGACCTCA-3' ${ }^{\prime}$, and GAPDH forward: $5^{\prime}$-TGACTTCAACAGCGACACCCA-3' and reverse: $5^{\prime}$-CACCCTGTTGCTGTAGCCAAA-3. Fold differences in expression were calculated using the comparative CT method by standardizing against GAPDH expression.

\section{Enzyme-Linked Immunosorbent Assay}

Quantikine enzyme-linked immunosorbent assay (ELISA) kits (MultiSciences, Biotech, Hangzhou, PR China) were used to detect IL-8 and TNF- $\alpha$. SZ95 sebocytes were seeded into 24-well plates $\left(10^{5}\right.$ cells/well $)$ and maintained in culture medium alone or treated with stimuli as indicated above. After incubation for $48 \mathrm{~h}$, culture supernatants were collected and centrifuged for removing cell detritus. Aliquots were stored at $-80{ }^{\circ} \mathrm{C}$ until use. TNF- $\alpha$ and IL- 8 protein levels were then determined according to the manufacturer's recommendation.

\section{Immunohistochemistry}

SZ95 sebocytes were grown on coverslips in 6-well plates $(5 \times$ $10^{5}$ cells/well) in culture medium alone or treated with stimuli as indicated above. Subsequently, the cells were fixed with $4 \%$ paraformaldehyde for $20 \mathrm{~min}$, incubated overnight at $4{ }^{\circ} \mathrm{C}$ with antiMyD88 antibody (ab133739, 1:500, Abcam), and then at room temperature with HRP goat anti-rabbit secondary antibody (ab97051, 1:500, Abcam) for $1 \mathrm{~h}$. The slices were then incubated in diaminobenzidine tetrachloride solution at room temperature for 5 min and counterstained with hematoxylin.

\section{Cell Transfection}

Human SZ95 sebocytes were seeded into 6-well plates at a density of $5 \times 10^{5} /$ well for $24 \mathrm{~h}$. AhR or control shRNA obtained from
GeneChem was transfected into the cells with Lipofectamine 2000 (Invitrogen) according to the manufacturer's instructions. After $48 \mathrm{~h}$ of transfection, the cells were treated as indicated above for further studies.

\section{Western Blot Analysis}

SZ95 sebocytes were seeded into 6 -well plates $\left(5 \times 10^{5}\right.$ cells/ well) in culture medium alone or treated with stimuli as indicated above, washed with PBS, and then lysed in cell lysis buffer (100 mM Tris-HCl, pH 6.8, 20\% glycerol, 4\% SDS, and 2\% mercaptoethanol) for $30 \mathrm{~min}$. Protein concentration was determined using a BCA protein assay kit (Thermo Fisher, Shanghai, PR China). Equal protein amounts were separated on SDS-PAGE and transferred to a nitrocellulose membrane. Blots were blocked in TBST $(20 \mathrm{mM}$ Tris- $\mathrm{HCl}$ at $\mathrm{pH} 7.4,150 \mathrm{~mm} \mathrm{NaCl}, 0.1 \%$ Tween-20) containing 5\% nonfat powder milk. The membranes were then incubated with antibodies against AhR (\#83200, 1:1000), phospho-p38MAPK (Thr 180/Tyr 182) (\#4511, 1:1000) and phospho-p65NF-kB (Ser536) (\#3033, 1:1000) (all Cell Signaling Technology, Danvers, MA, USA) overnight at $4{ }^{\circ} \mathrm{C}$. After three washes with PBS, the membranes were incubated with a secondary antibody (\#7074, 1: 2000) (Cell Signaling Technology) for $1 \mathrm{~h}$ at room temperature and washed again. The blots were developed by the chemiluminescence system ECL (Amersham, UK). Densitometry was performed by ImageJ.

\section{Statistical Analysis}

Values are presented as the mean of three represented independent experiments in each condition \pm SEM. A comparison of two groups was made with an unpaired, two-tailed Student's $t$ test. A comparison of multiple groups was made with analysis of variance (ANOVA). A two-way ANOVA was used when data with more than one factor were assessed. Differences were considered significant when: ${ }^{*} p<0.05,{ }^{* *} p<0.01,{ }^{* * *} p<0.001$, and ${ }^{* * * *} p<$ 0.0001 . All statistics were performed using GraphPad Prism version 6.0 software (San Diego, CA, USA). 

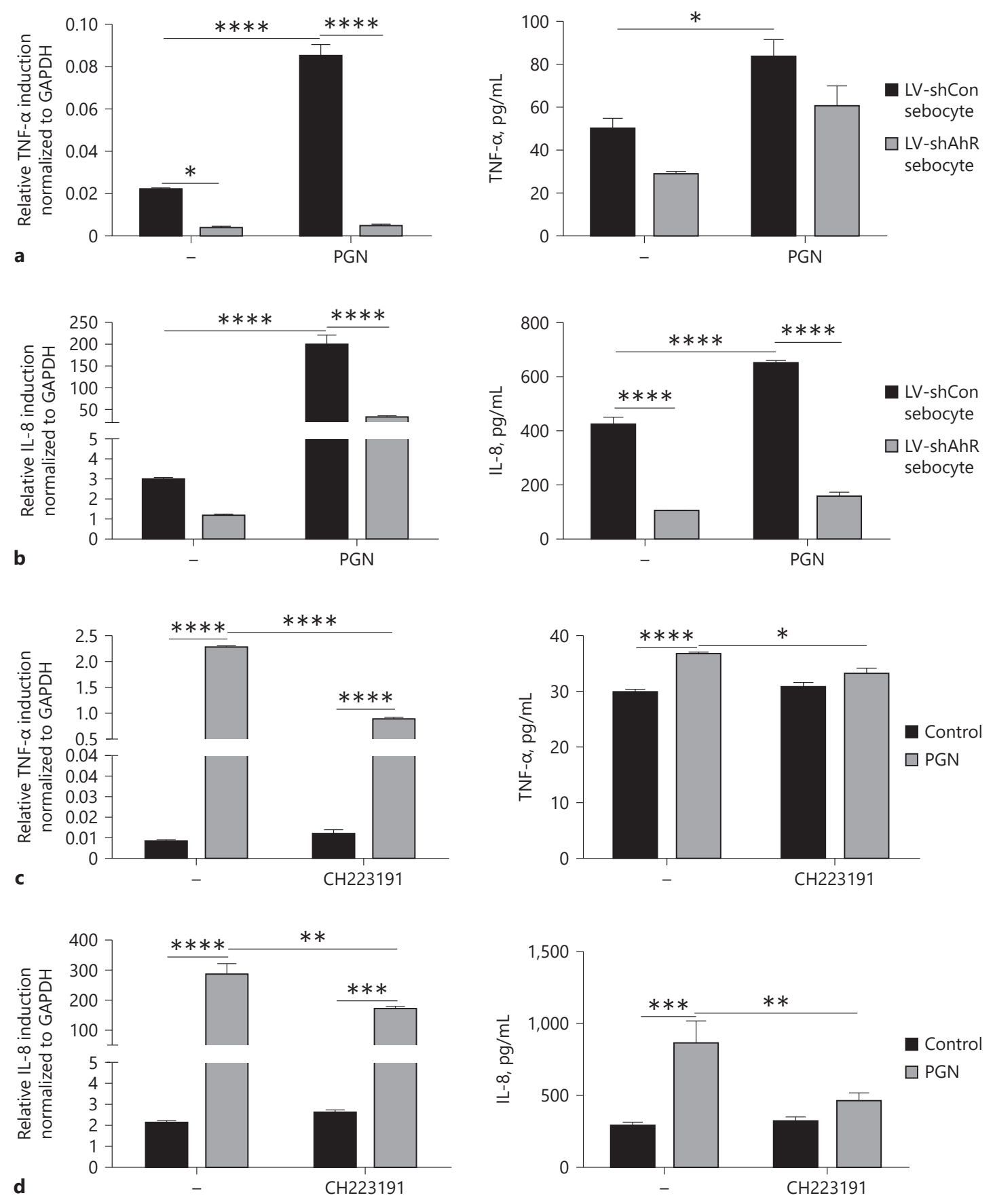

Fig. 2. PGN-induced secretion of TNF- $\alpha$ and IL- 8 is inhibited in AhR knockdown SZ95 sebocytes and CH223191-pretreated cells. Production of TNF- $\alpha$ (a) and IL-8 (b) in Lv-shAhR-transfected SZ95 sebocytes and Lv-shCon-transfected cells treated with PGN $(20 \mu \mathrm{g} / \mathrm{mL})$. Sebocytes were pretreated with the AhR antagonist $\mathrm{CH} 223191$ for $1 \mathrm{~h}$ in comparison with control cells (maintained in $0.1 \%$ DMSO), then the cells were treated with PGN $(20 \mu \mathrm{g} / \mathrm{ml})(\mathbf{c}$,

d), mRNA was extracted from cells treated with PGN for $3 \mathrm{~h}$. The culture supernatants were harvested from the cells treated with PGN for $48 \mathrm{~h}$. mRNA was detected by RT-PCR and relative results were normalized to GAPDH. Cytokines in culture supernatants were assayed by ELISA. Data are presented as the mean \pm SEM of at least 3 independent experiments. ${ }^{*} p<0.05$, ${ }^{* *} p<0.01$, ${ }^{* * *} p<$ $0.001,{ }^{* * * *} p<0.0001$. 


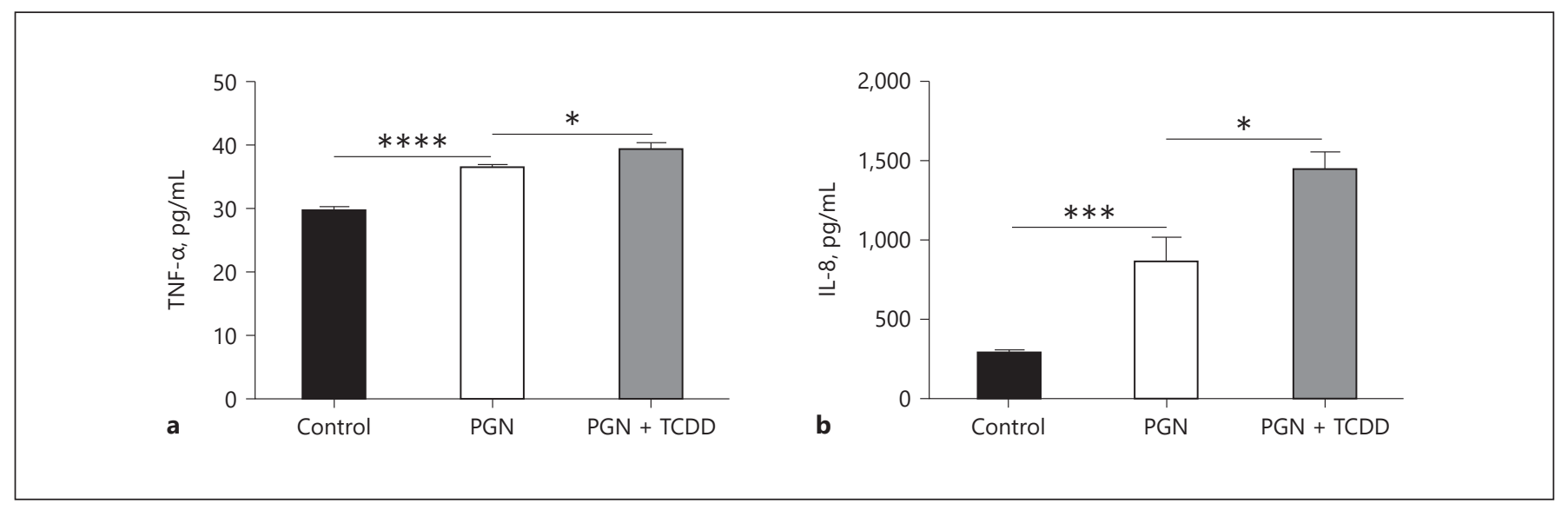

Fig. 3. Treatment with combined PGN and TCDD enhanced the release of TNF- $\alpha$ (a) and IL- 8 (b) from SZ95 sebocytes. TNF- $\alpha$ and IL-8 secretion from SZ95 sebocytes treated with PGN and TCDD was assessed at $48 \mathrm{~h}$. culture supernatants were assayed by ELISA. Data are presented as the mean \pm SEM of at least 3 independent experiments. ${ }^{*} p<0.05,{ }^{* * *} p<0.001,{ }^{* * * *} p<0.0001$.

\section{Results}

PGN-Induced TNF- $\alpha$ and IL-8 Production in Human SZ95 Sebocytes

First, we studied the effect of PGN $(20 \mu \mathrm{g} / \mathrm{mL})$, TCDD $(10 \mathrm{nM})$, and $\mathrm{CH} 223191(10 \mu \mathrm{M})$ on the viability of SZ95 sebocytes by the MTT assay after $48 \mathrm{~h}$ of treatment. As shown in Figure 1a, the investigated compounds had barely any effect on SZ95 sebocyte viability.

Second, human SZ95 sebocytes exposed to PGN (20 $\mu \mathrm{g} / \mathrm{mL}$ ) were investigated for cytokine synthesis. PGN stimulated cytokine mRNA levels at different time points. TNF- $\alpha$ mRNA expression increased 20-fold and IL-8 100 -fold at $3 \mathrm{~h}$ of treatment (Fig. 1b, c). Both mRNA levels were reduced over time up to $24 \mathrm{~h}$. These findings indicate an early stimulation of TNF- $\alpha$ and IL- 8 synthesis in SZ95 sebocytes by PGN.

\section{PGN-Induced Release of TNF- $\alpha$ and IL-8 is AhR} Dependent

To investigate if PGN induction of TNF- $\alpha$ and IL-8 synthesis by SZ95 sebocytes was followed by enhanced cytokine secretion, we investigated the PGN-induced secretion of TNF- $\alpha$ and IL-8 in both Lv-shAhR-transfected SZ95 sebocytes and SZ95 sebocytes treated with the AhR antagonist $\mathrm{CH} 223191$.

SZ95 sebocytes transfected with Lv-shCon and LvshAhR were exposed to PGN $(20 \mu \mathrm{g} / \mathrm{mL})$. mRNA expression of TNF- $\alpha$ and IL- 8 was detected by RT-PCR after 3 $\mathrm{h}$ of treatment and protein levels in supernatants were measured by ELISA after $48 \mathrm{~h}$ of treatment. Lv-shCon- and Lv-shAhR-transfected SZ95 sebocytes have been established and described in previous work [16]. In the present study, TNF- $\alpha$ and IL- 8 protein levels in the supernatants of Lv-shAhR-transfected cells were markedly reduced compared those of Lv-shCon-transfected SZ95 sebocytes (Fig. 2a, b).

To corroborate if PGN-induced secretion of proinflammatory cytokines was, indeed, AhR dependent, we repeated the experiments after pretreatment with the AhR antagonist CH223191 for $1 \mathrm{~h}$. SZ95 sebocytes were then treated with PGN alone or in the presence of $\mathrm{CH} 223191$ for 3 and $48 \mathrm{~h}$. TNF- $\alpha$ and IL-8 mRNA levels were both increased 100-fold under treatment with PGN in comparison with controls, while a $60 \%$ reduction in TNF- $\alpha$ mRNA levels and a 50\% reduction in IL-8 mRNA levels compared to PGN-treated cells were detected when cells were pretreated with $\mathrm{CH} 223191$ (Fig. 2c, d). Supernatant protein amounts confirmed the PGN-induced TNF- $\alpha$ and IL- 8 production. Pretreatment of SZ95 sebocytes with $\mathrm{CH} 223191$ reduced TNF- $\alpha$ and IL-8 protein levels (Fig. 2c, d).

Taken together, we provided evidence that the PGNinduced secretion of TNF- $\alpha$ and IL- 8 was, at least partially, AhR dependent in human SZ95 sebocytes.

\section{AhR Ligand TCDD Synergistically Enhanced the Production of TNF- $\alpha$ and IL-8 in PGN-Stimulated SZ95 Sebocytes}

SZ95 sebocytes were treated with PGN and the AhR agonist TCDD for $48 \mathrm{~h}$ and cytokine secretion was assessed by supernatant ELISA. The detected data suggest 


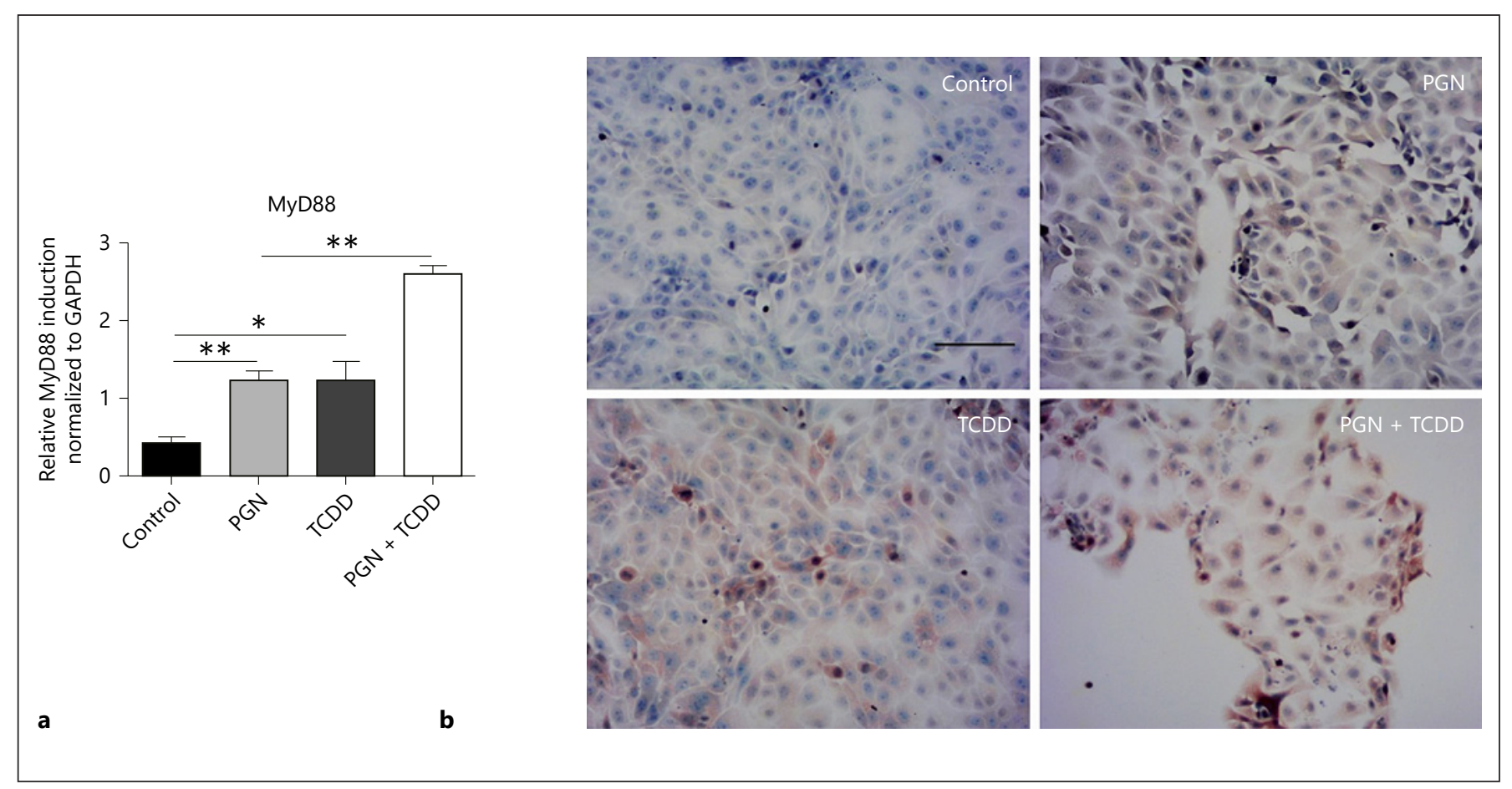

Fig. 4. MyD88 mRNA and protein expression in SZ95 sebocytes. a Cells were treated with PGN and TCDD for $3 \mathrm{~h}$. MyD88 mRNA expression was assessed by RT-PCR. Data are presented as the mean \pm SEM of at least 3 independent experiments. ${ }^{*} p<0.05$, ** $p<0.01$. b SZ95 sebocytes were treated with PGN and TCDD

that the combination treatment with TCDD and PGN resulted in an increase in secreted TNF- $\alpha$ and IL- 8 protein levels compared to cells treated with PGN alone (Fig. 3).

\section{TCDD Synergistically Enhanced the Expression of}

MyD88 in PGN-Treated SZ95 Sebocytes

PGN has been reported to be sensed by cell surface TLR2, resulting in secretion of inflammatory proteins [17]. Since we have shown that PGN-induced TNF- $\alpha$ and IL-8 secretion can be enhanced by TCDD in SZ95 sebocytes and MyD88 is a downstream gene of TLRs, we further studied the effect of TCDD on PGN-induced MyD88 expression. The presented data showed that PGN treatment of SZ95 sebocytes induced elevated levels of MyD88 mRNA and protein expression. Compared with cells treated with PGN, PGN + TCDD co-treatment of SZ95 sebocytes resulted in increased levels of MyD88 mRNA and protein (Fig. 4). for $24 \mathrm{~h}$. Anti-MyD88 immunocytology was used to evaluate localization and protein levels in SZ95 sebocytes. Representative expression patterns are shown of at least 3 independent experiments. Original magnification, $\times 100$. Scale bars, $200 \mu \mathrm{m}$.

\section{PGN Induces a Time-Dependent Phosphorylation of p38MAPK and p65NF- $\kappa B$ in Human SZ95 \\ Sebocytes}

NF- $\kappa B$ and MAPK are downstream genes in the innate immune signaling pathway and play a pivotal role in inflammatory and immune response. The Gram-positive bacterium C. acnes activates MAPKs in human keratinocytes [18]. We investigated, therefore, the effect of PGN (which is produced - among others - by the Gram-positive bacterium Staphylococcus aureus) on the expression of p38MAPK and p65NF- $\kappa$ B in SZ95 sebocytes. Incubation of SZ95 sebocytes with PGN $(20 \mu \mathrm{g} / \mathrm{mL})$ significantly induced phosphorylation of $\mathrm{p} 65 \mathrm{NF}-\mathrm{\kappa B}$ with peak at 15 min and p38MAPK with peak at $120 \mathrm{~min}$, and both exhibited a strong expression at $30 \mathrm{~min}$ (Fig. 5).

\section{AhR Inhibition Blocks the Ability of PGN to Stimulate Phosphorylation of $p 38 M A P K$ and $p 65 N F-\kappa B$ in SZ95 Sebocytes \\ To elucidate if the signaling pathways that are activat- ed by PGN are influenced by AhR expression level in}




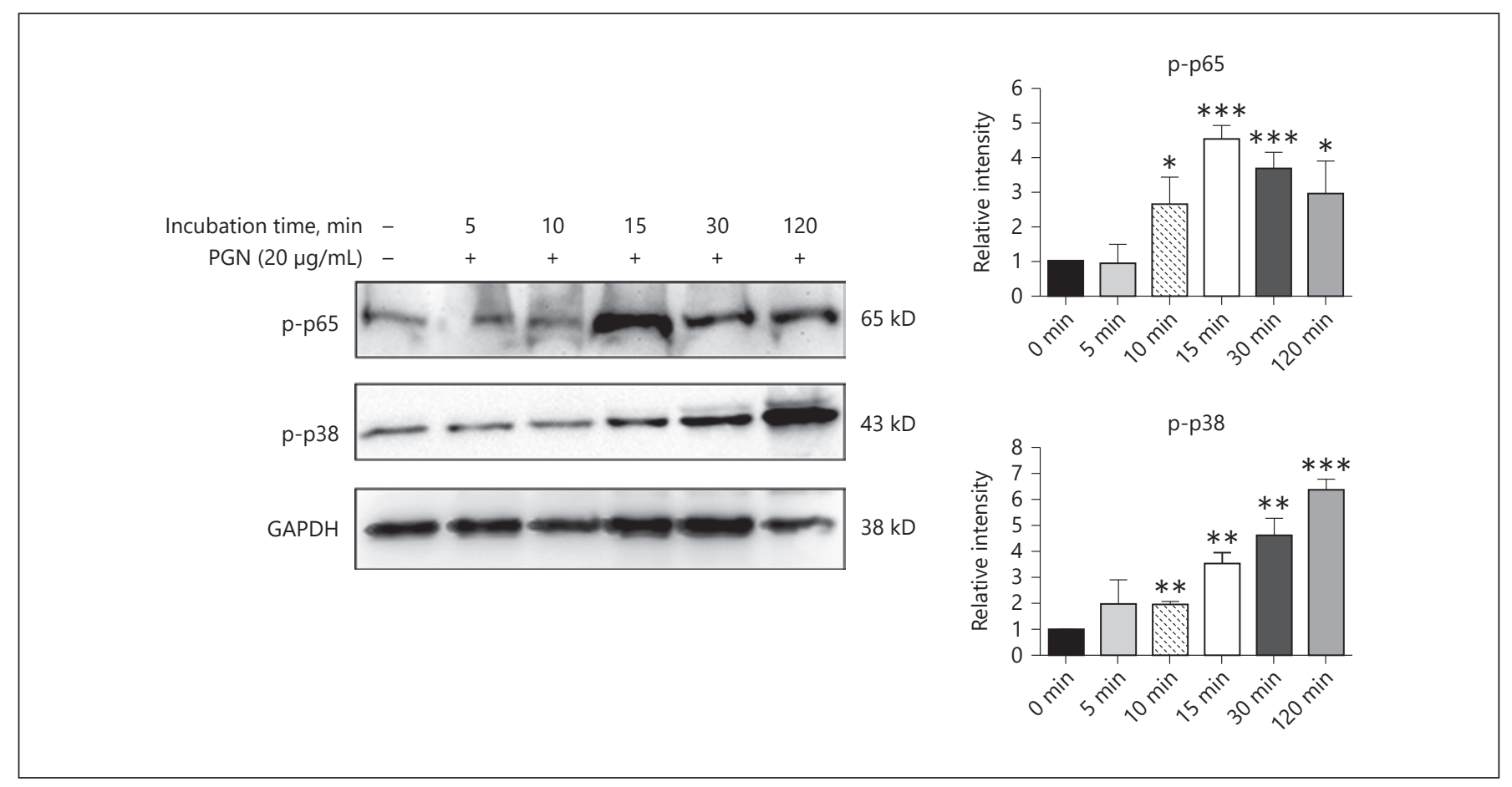

Fig. 5. PGN time dependently activates $\mathrm{p} 65 \mathrm{NF}-\kappa \mathrm{B}$ and p38MAPK. a Sebocytes were exposed to serum-free medium containing PGN $(20 \mu \mathrm{g} / \mathrm{mL})$ for different time periods $(0,5,10,15,30$, and $120 \mathrm{~min}$ ). Cell lysates from each sample were used for Western blot analyses performed as described in Materials and Methods with antibodies directed against p-p65NF- $\kappa$ B and p-p38MAPK; GAPDH was used as a loading control. The density of the phos- phorylated p38MAPK and p65NF- $\kappa$ B band was normalized to its loading control GAPDH. In $b$ and $c$, mean \pm SEM of relative arbitrary units of the bands from 3 immunoblots conducted in separate experiments are shown. ${ }^{*} p<0.05,{ }^{* *} p<0.01,{ }^{* * *} p<0.001$ versus $0 \mathrm{~min}$. A representative Western blot from 1 of 3 experiments is presented.
SZ95 sebocytes, we transfected SZ95 sebocytes with shRNAs, which specifically block AhR expression (AhR shRNA), or scrambled shRNA and subsequently treated the cells with PGN for $30 \mathrm{~min}$. First, we evaluated the efficiency of AhR shRNA in SZ95 sebocytes. Figure 6 shows that the transfection of SZ95 sebocytes with AhR shRNA significantly decreased AhR protein expression compared to the scrambled shRNA (negative control). Knockdown of AhR by AhR shRNA resulted in significantly decreased phosphorylation of $\mathrm{p} 38 \mathrm{MAPK}$ and $\mathrm{p} 65 \mathrm{NF}-\mathrm{kB}$ in comparison with cells transfected with a scrambled control (Fig. 6). Therefore, PGN, indeed, downregulated AhR expression in the scrambled control and also in the AhR knockout cells.

\section{TCDD Enhanced PGN-Induced Phosphorylation of p38MAPK and p65NF- $\kappa B$ in SZ95 Sebocytes}

To further understand the molecular basis of TCDD potentiation of PGN-induced activation of inflamma- tory signaling in SZ95 sebocytes, we next examined whether the phosphorylation of p38MAPK and p65NF$\kappa \mathrm{B}$ could be enhanced by TCDD. SZ95 sebocytes were pretreated with $\mathrm{CH} 223191$ for $1 \mathrm{~h}$ and subsequently treated with PGN and TCDD for $30 \mathrm{~min}$. Phosphorylation of $\mathrm{p} 65 \mathrm{NF}-\kappa \mathrm{B}$ and $\mathrm{p} 38 \mathrm{MAPK}$ was assessed by Western blot. As shown in Figure 7, treatment of SZ95 sebocytes with TCDD also caused a significant increase in phospho-p38 (p-p38) and p-p65 like that of PGN, whereas the combination treatment of PGN and TCDD induced a stronger effect than each compound alone. Pretreatment of cells with $\mathrm{CH} 223191$ significantly inhibited p-p38 and p-p65 levels, compared with PGN + TCDD-treated cells. These data indicate that the AhR agonist TCDD can enhance the phosphorylation of p65NF- $\kappa \mathrm{B}$ and $\mathrm{p} 38 \mathrm{MAPK}$ and the effect can be inhibited by an $A h R$ antagonist, facts which suggest $A h R$ to play a pivotal role in PGN-induced inflammatory response in human SZ95 sebocytes. 


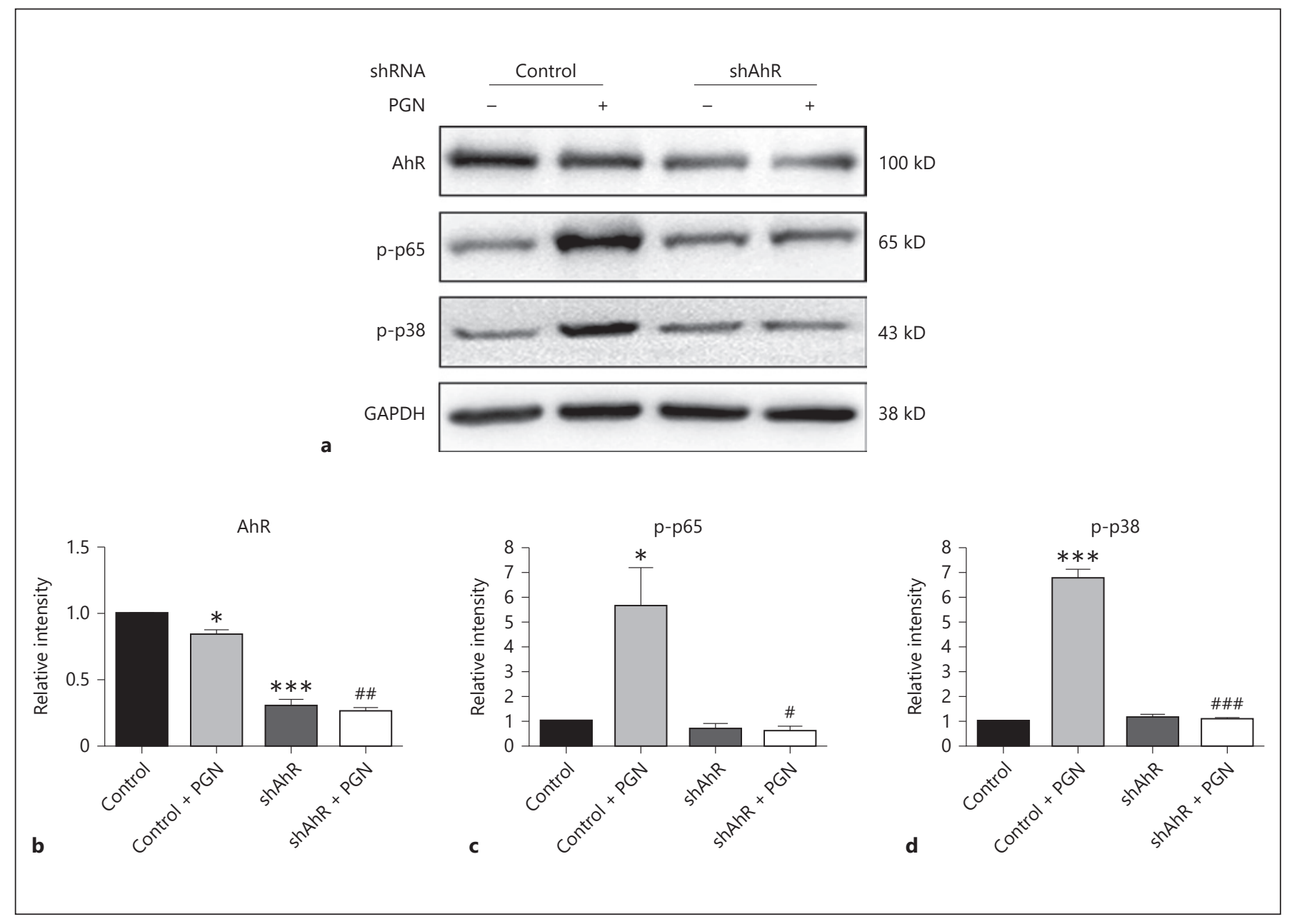

Fig. 6. AhR silencing through small hairpin RNA (shRNA) transfection suppressed PGN-induced phosphorylation of p65NF$\kappa B$ and p38MAPK. SZ95 sebocytes were transfected with AhR shRNA or scrambled shRNA. At $48 \mathrm{~h}$ after transfection, AhR protein expression and phosphorylation of p65 and p38 induced by PGN treatment for $30 \mathrm{~min}$ was evaluated using Western blotting. The density of AhR, phosphorylated p38MAPK and p65NF- $\kappa$ B band

\section{Discussion}

Acne is a chronic inflammatory disease of the sebaceous follicle [19]. Inflammation is a crucial factor at every stage of acne development. In inflammatory acne lesions, TNF- $\alpha$ and IL-8 expression is increased compared with the adjacent normal skin [20]. Cytokines, such as TNF- $\alpha$ and IL-8, propagate the inflammatory response by acting on endothelial cells to elaborate adhesion molecules (e.g. ICAM-1) to facilitate recruitment of professional inflammatory cells towards the skin lesions [21]. The transcription factor NF- $\kappa \mathrm{B}$ is activated in acne le- was normalized to its loading control GAPDH. Mean \pm SEM of relative arbitrary units of the bands from 3 immunoblots conducted in separate experiments are presented. ${ }^{*} p<0.05,{ }^{* * *} p<0.001$ versus the control. ${ }^{\#} p<0.05,{ }^{\#} p<0.01,{ }^{\# \# \#} p<0.001$ versus the control + PGN group. A representative Western blot from 1 of 3 experiments is shown. 
Fig. 7. TCDD treatment enhances PGNinduced phosphorylation of $\mathrm{p} 65 \mathrm{NF}-\mathrm{kB}$ and p38MAPK in SZ95 sebocytes. The cells were pretreated with the AhR antagonist $\mathrm{CH} 223191$ for $1 \mathrm{~h}$, then the $\mathrm{CH} 223191$ treated cells and controls were treated with PGN $(20 \mu \mathrm{g} / \mathrm{mL})$ and TCDD (10 nM) for 30 min. Cell lysates were used for Western blot analyses performed as described in Materials and Methods. The density of phosphorylated p38MAPK and p65NF- $\kappa$ B band was normalized to its loading control GAPDH. Mean \pm SEM of relative arbitrary units of the bands from 3 immunoblots conducted in separate experiments are presented. ${ }^{*} p<0.05,{ }^{* *} p<0.01,{ }^{* * *} p<0.001$, **** $p<0.0001$ versus the control. \#\# $p<$ 0.01 versus the PGN + TCDD group. A representative Western blot from 1 of 3 experiments is shown.

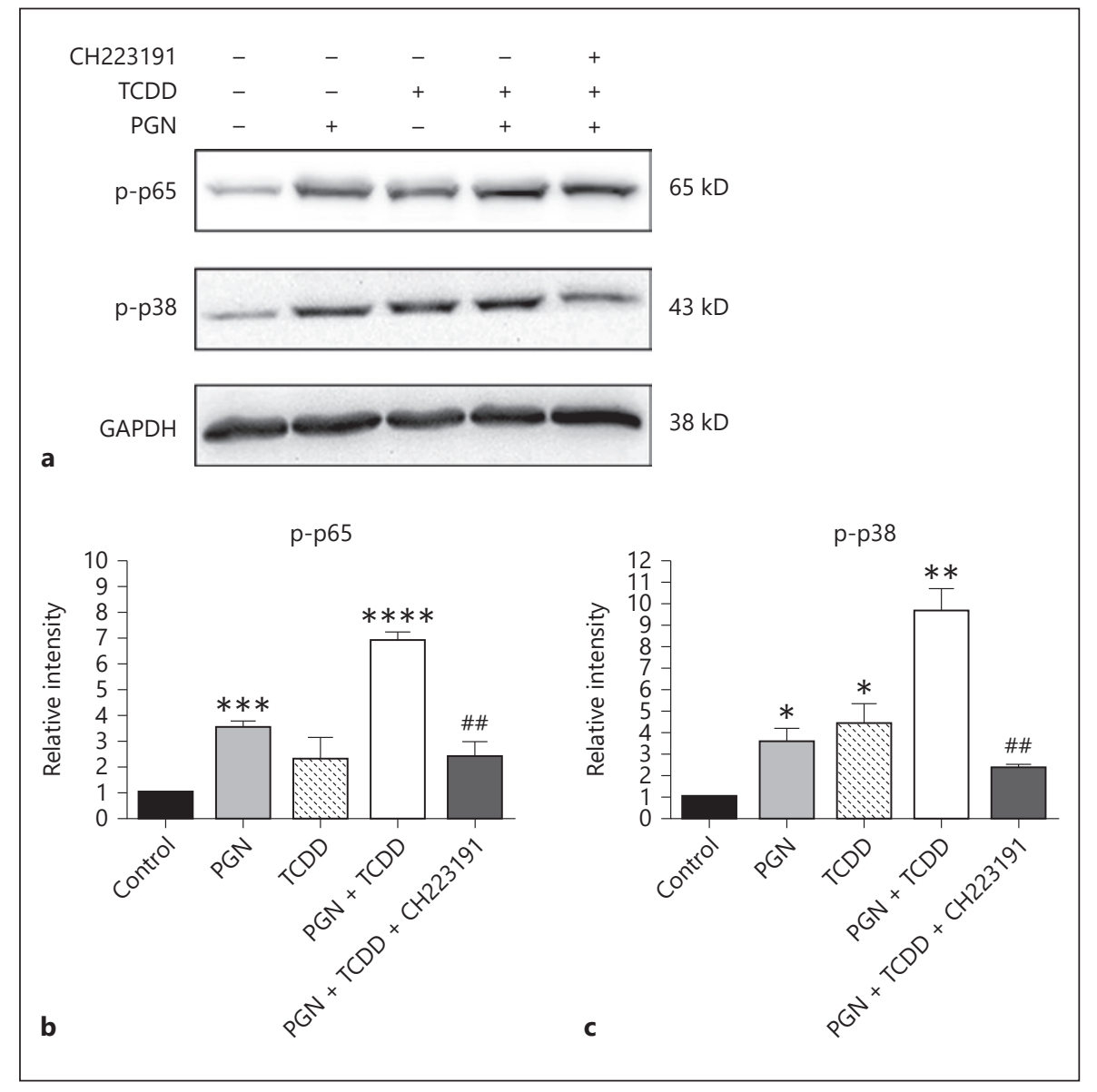

complex interplay between the immune system of the host and intestinal bacteria with detoxification mechanisms [23]. TCDD was shown to upregulate the expression and secretion of TNF- $\alpha$ and IL- 6 by increasing Akt phosphorylation and nuclear translocation of p $65 \mathrm{NF}-\kappa \mathrm{B}$ in cultured hepatic stellate cells [24] and TCDD induced MAPK phosphorylation in HepG2 cells [25].

The relationship between AhR and sebaceous glands is especially interesting, since AhR and CYP1A1 are both strongly expressed in human sebocytes and environmental contaminants TCDD and benzo(a)pyrene inhibit lipogenesis via switching sebocyte into keratinocyte differentiation $[16,26,27]$. Moreover, AhR may be involved in sebaceous gland homeostasis under both steady-state and stress (inflammatory) conditions [28].

In the current work, we used human SZ95 sebocytes to further elucidate the role of AhR in the inflammation process. Interestingly, a PGN-induced release of TNF- $\alpha$ and IL-8 was detected to be - at least partially - AhR dependent, as shown in both AhR knockdown SZ95 sebocytes and by the AhR antagonist $\mathrm{CH} 223191$ administration. The AhR agonist TCDD enhanced the PGN-induced TNF- $\alpha$ and IL- 8 cellular secretion.

Upon pathogen-associated molecular pattern recognition, TLRs are activated and subsequently transduce signals from the cell membrane to the nucleus [29]. Currently, two main branches of the TLR signal transduction pathway have been identified, namely the MyD88- and the TRIF-dependent pathways [30]. The principal signaling pathways downstream to MyD88 are the NF- $\kappa B$ and MAPK-AP-1 pathways [31]. We demonstrated that PGN-induced MyD88 mRNA levels and protein expression in SZ95 sebocytes as well as TCDD strengthen this effect (Fig. 4). Our results also showed a PGN-induced phosphorylation of p65NF- $\kappa$ B and p38MAPK in SZ95 sebocytes, whereas p65 and p38 phosphorylation could be disrupted by knocking down AhR. Moreover, TCDD exhibited a synergistic effect on the PGN-induced phosphorylation of p65 and p38, which could be inhibited by the AhR antagonist CH223191. Taken together, these ob- 
servations clearly indicate that $\mathrm{AhR}$ is critically involved in PGN-induced TNF- $\alpha$ and IL-8 synthesis in human SZ95 sebocytes via MyD88-p38MAPK/p65NF- $\kappa B$ signal transduction pathways. Since this project focused on the TLR and PGN downstream pathway, it apparently represents a classic TLR2 agonistic mode of action. The investigation of AhR-associated influence of TLR2 expression is the target of future studies.

As shown in Figure 6, PGN, indeed, downregulated AhR expression in the scrambled control and also in the AhR knockout cells. PGN is a classic ligand of TLR2, but in our work it could also directly influence the AhR signal pathway. Downregulation of AhR expression in SZ95 sebocytes at the protein level has been proven in our previous studies under treatment with the typical AhR agonists TCDD [26] and benzo(a)pyrene [16]. On the other hand, this study has shown that PGN activates the AhR signal pathway, whereas the exact molecular mechanism still needs to be studied in the future.
In recent years, increasing evidence indicates a link between acne and exposure to environmental pollutants through unclear mechanisms [32]. Our data elaborate the molecular mechanisms of AhR - a transcription factor that senses environmental stimuli to regulate innate immunity in human SZ95 sebocytes - and provide a new insight into the association between environment pollution and regulation of innate immunity in acne.

\section{Acknowledgments}

This work was supported by the National Natural Science Foundation of China (grant No. 81472894 and 81502718).

\section{Disclosure Statement}

The authors declare that they have no conflict of interest.

\section{References}

1 Takeuchi O, Akira S: Pattern recognition receptors and inflammation. Cell 2010;140: 805-820.

- Selway JL, Kurczab T, Kealey T, Langlands K: Toll-like receptor 2 activation and comedogenesis: implications for the pathogenesis of acne. BMC Dermatol 2013;13:10.

$\checkmark 3$ Zouboulis CC: Sebaceous gland receptors. Dermatoendocrinol 2009;1:77-80.

-4 Huang YC, Yang CH, Li TT, Zouboulis CC, Hsu HC: Cell-free extracts of Propionibacterium acnes stimulate cytokine production through activation of p38 MAPK and Tolllike receptor in SZ95 sebocytes. Life Sci 2015; 139:123-131.

5 Alestas T, Ganceviciene R, Fimmel S, MullerDecker K, Zouboulis CC: Enzymes involved in the biosynthesis of leukotriene B4 and prostaglandin E2 are active in sebaceous glands. J Mol Med 2006;84:75-87.

6 Nagy I, Pivarcsi A, Kis K, Koreck A, Bodai L, McDowell A, Seltmann H, Patrick S, Zouboulis CC, Kemeny L: Propionibacterium acnes and lipopolysaccharide induce the expression of antimicrobial peptides and proinflammatory cytokines/chemokines in human sebocytes. Microbes Infect 2006;8:2195-2205.

7 Iinuma K, Sato T, Akimoto N, Noguchi N, Sasatsu M, Nishijima S, Kurokawa I, Ito A: Involvement of Propionibacterium acnes in the augmentation of lipogenesis in hamster sebaceous glands in vivo and in vitro. J Investig Dermatol 2009;129:2113-2119.
8 Panteleyev AA, Bickers DR: Dioxin-induced chloracne - reconstructing the cellular and molecular mechanisms of a classic environmental disease. Exp Dermatol 2006;15:705-730.

-9 Nguyen NT, Hanieh H, Nakahama T, Kishimoto $\mathrm{T}$ : The roles of aryl hydrocarbon receptor in immune responses. Int Immunol 2013; 25:335-343.

10 Kung MH, Yukata K, O’Keefe RJ, Zuscik MJ: Aryl hydrocarbon receptor-mediated impairment of chondrogenesis and fracture healing by cigarette smoke and benzo( $\alpha$ )pyrene. J Cell Physiol 2012;227:1062-1070.

-11 Kado S, Chang WLW, Chi AN, Wolny M, Shepherd DM, Vogel CFA: Aryl hydrocarbon receptor signaling modifies Toll-like receptor-regulated responses in human dendritic cells. Arch Toxicol 2017;91:2209-2221.

12 Kimura A, Naka T, Nakahama T, Chinen I, Masuda K, Nohara K, Fujii-Kuriyama Y, Kishimoto T: Aryl hydrocarbon receptor in combination with Statl regulates LPS-induced inflammatory responses. J Exp Med 2009;206:2027-2035.

13 Tian Y: Ah receptor and NF- $\mathrm{BB}$ interplay on the stage of epigenome. Biochem Pharmacol 2009;77:670-680.

14 Vondracek J, Umannova L, Machala M: Interactions of the aryl hydrocarbon receptor with inflammatory mediators: beyond CYP1A regulation. Curr Drug Metab 2011;12:89-103.
15 Zouboulis CC, Seltmann H, Neitzel H, Orfanos CE: Establishment and characterization of an immortalized human sebaceous gland cell line (SZ95). J Investig Dermatol 1999;113: 1011-1020.

$16 \mathrm{Hu}$ T, Pan Z, Yu Q, Mo X, Song N, Yan M, Zouboulis CC, Xia L, Ju Q: Benzo(a)pyrene induces interleukin (IL)-6 production and reduces lipid synthesis in human SZ95 sebocytes via the aryl hydrocarbon receptor signaling pathway. Environ Toxicol Pharmacol 2016;43:54-60.

17 Oliveira-Nascimento L, Massari P, Wetzler LM: The role of TLR2 in infection and immunity. Front Immunol 2012;3:79.

18 Li WH, Zhang L, Lyte P, Rodriguez K, Cavender D, Southall MD: p38 MAP kinase inhibition reduces Propionibacterium acnes-induced inflammation in vitro. Dermatol Ther 2015;5:53-66.

19 Moradi Tuchayi S, Makrantonaki E, Ganceviciene R, Dessinioti C, Feldman SR, Zouboulis CC: Acne vulgaris. Nat Rev Dis Primers 2015; $1: 15029$.

20 Kang S, Cho S, Chung JH, Hammerberg C, Fisher GJ, Voorhees JJ: Inflammation and extracellular matrix degradation mediated by activated transcription factors nuclear factor$\kappa \mathrm{B}$ and activator protein-1 in inflammatory acne lesions in vivo. Am J Pathol 2005; 166 : 1691-1699. 
21 Collins T, Read MA, Neish AS, Whitley MZ, Thanos D, Maniatis T: Transcriptional regulation of endothelial cell adhesion molecules: $\mathrm{NF}-\mathrm{KB}$ and cytokine-inducible enhancers. FASEB J 1995;9:899-909.

22 Esser C: Biology and function of the aryl hydrocarbon receptor: report of an international and interdisciplinary conference. Arch Toxicol 2012;86:1323-1329.

23 Do KN, Fink LN, Jensen TE, Gautier L, Parlesak A: TLR2 controls intestinal carcinogen detoxication by CYP1A1. PLoS One 2012; 7:e32309.

24 Han M, Liu X, Liu S, Su G, Fan X, Chen J, Yuan Q, Xu G: 2,3,7,8-Tetrachlorodibenzo- $p$ dioxin (TCDD) induces hepatic stellate cell (HSC) activation and liver fibrosis in C57BL6 mouse via activating Akt and NF- $\mathrm{kB}$ signaling pathways. Toxicol Lett 2017;273:10-19.
5 Palanisamy K, Krishnaswamy R, Paramasivan P, Chih-Yang H, Vishwanadha VP: Eicosapentaenoic acid prevents TCDD-induced oxidative stress and inflammatory response by modulating MAP kinases and redox-sensitive transcription factors. Br J Pharmacol 2015;172:4726-4740.

26 Ju Q, Fimmel S, Hinz N, Stahlmann R, Xia L, Zouboulis CC: $2,3,7,8$-Tetrachlorodibenzo- $p$ dioxin alters sebaceous gland cell differentiation in vitro. Exp Dermatol 2011;20:320-325

$7 \mathrm{Hu}$ T, Wang D, Yu Q, Li L, Mo X, Pan Z, Zouboulis CC, Peng L, Xia L, Ju Q: Aryl hydrocarbon receptor negatively regulates lipid synthesis and involves in cell differentiation of SZ95 sebocytes in vitro. Chem Biol Interact 2016;258:52-58.
28 Bock KW: Toward elucidation of dioxin-mediated chloracne and $\mathrm{Ah}$ receptor functions. Biochem Pharmacol 2016;112:1-5.

29 Kawai T, Akira S: TLR signaling. Cell Death Differ 2006;13:816-825.

30 Adachi O, Kawai T, Takeda K, Matsumoto M, Tsutsui H, Sakagami M, Nakanishi K, Akira S: Targeted disruption of the MyD88 gene results in loss of IL-1 - and IL-18-mediated function. Immunity 1998;9:143-150.

31 Kawai T, Akira S: Toll-like receptor downstream signaling. Arthritis Res Ther 2005;7: 12-19.

32 Krutmann J, Moyal D, Liu W, Kandahari S, Lee GS, Nopadon N, Xiang LF, Seite S: Pollution and acne: is there a link? Clin Cosmet Investig Dermatol 2017;10:199-204. 